\title{
Guías chilenas de práctica clínica de tratamiento del tabaquismo, 2017
}

\author{
Smoking Cessation: Chilean Clinical Practice Guidelines
}

Para el control efectivo de la epidemia de tabaquismo, se requiere de la acción organizada de los múltiples actores que participan en las decisiones de políticas públicas en salud. El Convenio Marco para el Control del Tabaco (CMCT) así lo establece en sus consideraciones iniciales, estimulando al trabajo integrado e involucrando, entre otros actores, a los denominados "órganos de las profesiones de la salud". El mismo documento, señala la conveniencia que los estados adopten, además de medidas regulatorias para restringir la publicidad, comercialización y consumo de los productos del tabaco, medidas complementarias para reducir su consumo ${ }^{1}$.

Han sido estas las motivaciones para emprender el desafio de avanzar en la proposición de la primera guía clínica chilena orientada a abordar la cesación del consumo de tabaco. Existe la necesidad de complementar la estrategia nacional regulatoria, propiciada por la ley de tabaco vigente, con acciones que aborden el apoyo a miles de chilenos que requieren de ayuda profesional para concretar el abandono del consumo de tabaco. A la fecha, se trata esta de una tarea pendiente no incorporada en la política pública correspondiente ${ }^{2}$. Al compromiso de la Sociedad Chilena de Enfermedades Respiratorias (SER), se ha sumado la energía y entusiasmo de otras sociedades cientificas médicas y grupos de profesionales, consolidando una fuerza de tarea que se abocó a la producción de dicha guía clínica. Este grupo de trabajo estuvo conformado, además de la SER, por la Sociedad Chilena de Cardiología y la Sociedad Chilena de Endocrinología y Diabetes, con un conjunto de médicos de diferentes especialidades (salud pública, medicina familiar, psiquiatría, pediatría, oncología, medicina interna, enfermedades respiratorias, diabetes y endocrinología, nutrición), psicólogos, kinesiólogos y enfermeras. Esa fuerza de tarea, es inédita en su conformación y se alinea con las orientaciones del CMCT en cuanto a su amplia representación. A la conveniencia técnica de asumir el compromiso de apoyo en cesación, se agrega el componente ético, expresado por la responsabilidad de asumir como país la introducción de este componente, para el cual ya se dispone de evidencia sólida respecto de los beneficios obtenidos de intervenciones clínicas adecuadas en apoyo a la cesación.

El desarrollo técnico utilizado en la confección de dicha guía, se basó en los lineamientos de la Metodología GRADE (Grading of Recommendations Assessment, Development and Evaluation), la que genera una sintesis de la evidencia obtenida, incorporando también elementos como valores, preferencias, beneficios, efectos indeseados y factibilidad de implementar intervenciones. Cabe hacer notar que esta metodología ha sido incorporada oficialmente por instituciones relevantes en materia de control del tabaquismo, como son la Organización Mundial de la Salud (OMS), National Cancer Institute (NCI), U.S. Preventive Services Task Force (USPSTF), Sociedad Americana del Tórax (ATS) y la Sociedad Europea de Enfermedades Respiratorias (ERS) ${ }^{3,4,5}$. Localmente, el Ministerio de Salud está ya utilizando esta metodología en el desarrollo de instrumentos de esta naturaleza ${ }^{6}$.

Esta metodología ofrece ventajas sobre otros sistemas de graduación de recomendaciones, ya que fue desarrollado por un grupo amplio y representativo de elaboradores de guías a nivel internacional, posee una clara separación entre la calidad de la evidencia y la fuerza de la recomendación, además de poseer una evaluación explícita de la importancia de los desenlaces para estrategias alternativas. También tiene un sistema de evaluación de la calidad de la evidencia claro y explícito, realizando las recomendaciones a través de un proceso trasparente. Además toma de forma explícita los valores y preferencias de los pacientes, y la interpretación de la fuerza de la recomendación para los clínicos, pacientes y tomadores de decisión es clara y pragmática ${ }^{7}$. Existe evidencia que sugiere que las Guías de Práctica Clínica realizadas rigurosamente, logran transferir el conocimiento y mejorar la calidad de la salud en indicadores de estructura, proceso y resultado ${ }^{8}$. 
En el proceso del sistema GRADE se realizan preguntas que delimitan claramente el alcance de la guía en desarrollo. En la actual guía se definieron 6 preguntas por consenso del panel, tres de ellas relativas a intervenciones no farmacológicas y tres que consideran intervenciones farmacológicas de tratamiento del tabaquismo. 1.- ¿Se debe utilizar la consejería breve versus no intervención en fumadores? 2.- ¿Se deben utilizar intervenciones basadas en telefonía móvil versus no intervención en fumadores? 3.- ¿Se deben utilizar intervenciones basadas en mensajes de texto versus no intervención en fumadores? 4.- ¿Se debe utilizar terapia de reemplazo nicotínico sobre no intervención en individuos dependientes de nicotina con intención de dejar de fumar? 5.- ¿Se debe utilizar Bupropión sobre no intervención en individuos dependientes de nicotina con intención de dejar de fumar? 6.- ¿Se debe utilizar Vareniclina sobre no intervención en individuos dependientes de nicotina con intención de dejar de fumar?

Para cada una de las preguntas, se elaboró una tabla utilizando el software GDT GRADEpro (https://gradepro.org/). Cada tabla resume la evidencia y su certeza, además de las recomendaciones. Este programa es una herramienta que facilita el desarrollo de recomendaciones y su actualización cuando aparece nueva evidencia. En consecuencia, la metodología utilizada admite la opción de incorporar en forma progresiva nuevas recomendaciones y también, la revisión actualizada de las recomendaciones realizadas.

\section{Descripción breve del problema y su cuantificación}

La persistente alta prevalencia en el consumo de tabaco es un fenómeno que ha caracterizado a la epidemiología del tabaquismo en Chile. Esto se traduce en una elevada carga de enfermedad atribuible al consumo directo y a la exposición al humo ambiental de tabaco, con una estimación de más de 16.000 muertes anuales atribuibles a dicho consumo.

Resulta promisoria la tendencia observada en la Segunda Encuesta Nacional de Salud ${ }^{9}$ (ENS 200910), medición efectuada con posterioridad al endurecimiento de las medidas de control incluidas en la modificación (2006) de la ley respectiva*. Dicha encuesta mostró una reducción en la prevalencia en varones e incremento en las tasas de población exfumadora (11,1\% a 18\% entre 2003 y 2010).

Por su parte, la tendencia de la serie de estudios del SENDA ${ }^{10}$ en población escolar chilena, muestra para los mismos años una sistemática reducción de la prevalencia de consumo en el último mes (desde 51,6\% a 40\% entre los años 2001-2015). Y por último la encuesta mundial de tabaquismo en jóvenes (EMTJ), Chile 2016 ${ }^{10}$, evidencia un cambio significativo (desde 34,2 a 17,2\% en la prevalencia de fumadores actuales (que fumaron cigarrillo en algún momento de los últimos 30 días) en la población de 13 a 15 años de la Región Metropolitana, también hubo disminución significativa en el consumo en el resto de las regiones evaluadas en este estudio: Arica y Parinacota, Tarapacá, Valparaíso y Bío Bío.

Recientemente, en un análisis agrupado de países, se ha comunicado una reducción de 6,6\% en la prevalencia de fumadores actuales, comportamiento estadísticamente relacionado con la sucesiva implementación de directrices del CMCT ${ }^{11}$. Cabe preguntarse cuál habría sido dicha tendencia de haber incorporado Chile el componente de cesación en sus políticas nacionales de salud.

En espera de los resultados de la Tercera Encuesta Nacional de Salud (ENS) 2016, son también promisorios los resultados de la Encuesta Nacional de Calidad de Vida (ENCAVI) 2015-16 ${ }^{12}$, la que muestra reducción de $40 \%$ a $30 \%$ en la prevalencia mensual de consumo y reducción en la exposición a humo ambiental de tabaco en el hogar, desde 51,5\% a 28,1\%, ambas mediciones efectuadas en los años 2000 y 2015-16.

Por otra parte, y en base a los antecedentes de la ENS 2009-10, se estima que el 25\% de los fumadores diarios varones de 15 o más años, son dependientes de la nicotina. Para las mujeres, esta estimación alcanza al 22\%. Esto se traduce en una estimación nacional de dependencia a la nicotina para población de 15 años o más, de 8\% y de 5,8\% en varones y mujeres chilenas, respectivamente.

\section{El fumador, sujeto de intervenciones en esta guía clínica}

Las personas fumadoras tienen un mayor riesgo de enfermar y morir, a consecuencia de esas en- 
fermedades, que las personas que no fuman, o que los exfumadores. Las evidencias de la literatura médica se han acrecentado desde hace más de 50 años y el Informe del Cirujano General de EE.UU del año $2014^{13}$ resume la información disponible. Por otra parte, dejar de fumar tiene beneficios para la salud y mejora la sobrevida. Es así como el clásico trabajo de Doll y cols. ${ }^{14}$, muestra que en la cohorte de médicos ingleses seguida por 50 años, los fumadores mueren en promedio 10 años antes que los no fumadores y que la cesación del consumo de tabaco a los 60, 50, 40 o 30 años de edad aumenta la expectativa de vida en 3, 6, 9 o 10 años respectivamente.

En nuestro país Pichón Riviere y cols. ${ }^{15}$, muestran que el tabaquismo es responsable de una importante cantidad de muertes, enfermedad y costos sanitarios. La mayor carga de enfermedad está dada por la EPOC (Enfermedad Pulmonar Obstructiva Crónica), diversos tipos de cáncer y enfermedades cardiovasculares. Su impacto en la mortalidad y en la calidad de vida es responsable en forma directa de la pérdida de 428.588 años de vida y causa el 18,5\% de todas las muertes que se producen en el país cada año, muertes que podrían ser evitadas.

Por lo tanto ayudar a que las personas dejen de fumar es una estrategia esencial en las politicas de salud pública para el control del consumo de tabaco y un imperativo ético para tratar a los fumadores cuando consultan en los servicios de salud. Lamentablemente en muchos países, y Chile no es la excepción, no se le ha prestado a esta política todos los esfuerzos y los recursos necesarios, ya que además es la estrategia que permitiría en forma más rápida disminuir la mortalidad, considerando que las otras estrategias, como las de prevención primaria, tienen su efecto en el más largo plazo.

Es por esto que el CMCT de la OMS en su artículo 14 señala: "Cada Parte elaborará y difundirá directrices apropiadas, completas e integradas, basadas en pruebas cientificas y en las mejores prácticas, teniendo presente las circunstancias y prioridades nacionales, y adoptará medidas eficaces para promover el abandono del consumo de tabaco y el tratamiento adecuado de la dependencia del tabaco".

Lo anterior, mediante el diseño de "programas eficaces de promoción del abandono del consumo de tabaco e incorporando el diagnóstico y el tratamiento de la dependencia del tabaco y servicios de asesoramiento sobre el abandono del tabaco".

Por otra parte, las políticas de promoción de la cesación tabáquica y tratamiento de la dependencia del tabaquismo son costo-efectivas y tienen un alto impacto sanitario en términos de reducción de la mortalidad. En Chile esto no se ha abordado en la forma de un programa de cobertura nacional, lo que sin duda es un aspecto pendiente por parte del Ministerio de Salud, por lo cual además se está incumpliendo el CMCT.

Esta Guía de Práctica Clínica de Tratamiento del Tabaquismo de nuestro país, orientadora de la acción del personal de salud, es una herramienta esencial en el control del consumo de tabaco. Dentro de las acciones complementarias a éstas y que las sociedades cientifico-profesionales deben considerar, se cuentan ${ }^{16}$ :

- La inclusión de temáticas sobre tabaco en los programas de estudios superiores, especialmente en carreras de la salud, dado el papel fundamental de los profesionales en la prevención $1^{\text {aria }}$ y $2^{\text {aria }}$.

- El desarrollo de investigación local sobre las causas, mecanismos de daño, efectos y consecuencias sociales, de salud y económicas del consumo de tabaco.

La aplicación y uso de esta guía tiene como destinatarios a profesionales de salud que se desempeñan en cualquier punto de atención en las redes de servicios públicos o privados, incluyendo el ámbito hospitalario. Debe recordarse que la Joint Commission ${ }^{17}$ requiere para efectos de acreditación, que hospitales y clínicas evalúen la condición de fumadores de sus pacientes y documenten la oferta de consejería y/o terapia farmacológica y el seguimiento de dichas acciones, como un criterio más de calidad de atención.

La SER espera que este instrumento contribuya a potenciar la lucha contra el consumo de tabaco, otorgando más y mejores oportunidades de cesación a la población chilena. Para el logro del impacto esperado de estas acciones es indispensable el compromiso rector del sistema de salud chileno, en 
cuanto a incluir y considerar la cesación como un elemento indispensable en el control del consumo de tabaco, aportando los recursos y el financiamiento necesario para una política pública nacional.

\author{
Dr. Gonzalo Valdivia $C$. \\ Dpto. de Salud Pública. Facultad de Medicina \\ Pontificia Universidad Católica de Chile \\ Email:valdivia@med.puc.cl
}

\author{
Dr. Sergio Bello $S$. \\ Programa de Tabaquismo \\ Instituto Nacional del Tórax \\ Email:sbello@torax.cl \\ Integrantes Comisión de Tabaco
}

Sociedad Chilena de Enfermedades Respiratorias

\section{Bibliografía}

1.- Organización Mundial de la Salud, Ginebra, Suiza. Convenio Marco de la OMS para el Control del Tabaco. OMS 2003, reimpresión actualizada, 2004, 2005. ISBN 924359101 .

2.- Informe sobre el Control del Tabaco en la Región de las Américas. A 10 años del Convenio Marco de la Organización Mundial de la Salud para el Control del Tabaco. Washington, DC: OPS, 2016. Página 29.

3.- KAVANAGH BP. (2009). The GRADE System for Rating Clinical Guidelines. PLoS Medicine, 6(9), e1000094. http://doi.org/10.1371/journal.pmed.1000094

4.- WHO Recommendations for the Prevention and Management of Tobacco Use and Second-Hand Smoke Exposure in Pregnancy. Geneva: World Health Organization; 2013. Executive Summary. Disponible en: https://www. ncbi.nlm.nih.gov/books/NBK190297/

5.- http://canadiantaskforce.ca/wp-content/uploads/2017/02/ Tobacco-Systematic-Review_FINAL.pdf

6.- Subsecretaría de Salud Pública. División de Prevención y Control de Enfermedades. Departamento Secretaria AUGE y de Coordinación Evidencia y Metodológica. Desarrollo de Guías de Práctica Clínica. Manual Metodológico. Septiembre 2014.

7.- GUYATT GH, OXMAN AD, VIST GE, KUNZ R, FALCK-YTTER Y, ALONSO-COELLO P, et al. GRADE: an emerging consensus on rating quality of evidence and strength of recommendations. The GRADE working group. BMJ 2008; 336; 924-6.

8.- Ministerio de Salud, Segunda Encuesta nacional de Salud 2009-10. Disponible en: http://web.minsal.cl/ portal/url/item/bcb03d7bc28b64dfe040010165012d23. pdf Consultada: 07/mayo, 2017.

9.- Servicio Nacional para la Prevención y Rehabilitación del Consumo de Drogas y Alcohol (SENDA). Décimo primer estudio nacional de drogas en población escolar (2015).Observatorio Chileno de Drogas. Disponible en: http://www.senda.gob.cl/media/estudios/PE/Informe $\% 20$ Nacional $\% 20$ Final $\% 20 \mathrm{ENPE} \% 202015$.pdf
Consultado: 7/05/2017.

10.- Encuesta Mundial de Tabaquismo en Jóvenes (EMTJ) 2016, disponible en http//reb.minsal.cl/wp_content/ uploods/2017/10/EMTJ2016_17_10_2017_UF pdf

11.- GRAVELY S, GIOVINO GA, CRAIG L, COMMAR A, D'ESPAIGNET ET, SCHOTTE K, et al. Implementation of key demand-reduction measures of the WHO Framework Convention on Tobacco Control and change in smoking prevalence in 126 countries: an association study. The Lancet Public Health; 2017: 2: e166-e174. Disponible en : http://thelancet.com/pdfs/journals/lanpub/ PIIS2468-2667(17)30045-2.pdf Consultado: 7/05/2017.

12.- Encuesta de Calidad de Vida y Salud (ENCAVI), 2015 16. División de Planificación Sanitaria Departamento de Epidemiología Unidad de Vigilancia de Enfermedades No Transmisibles. Ministerio de Salud, Febrero 2017.

13.- U.S. Department of Health and Human Services. The Health Consequences of Smoking-50 Years of Progress: A Report of the Surgeon General. Atlanta, GA: U.S. Department of Health and Human Services, Centers for Disease Control and Prevention, National Center for Chronic Disease Prevention and Health Promotion, Office on Smoking and Health, 2014.

14.- DOLL R, PETO R, BOREHAM J, SUTHERLAND I. Mortality in relation to smoking: 50 years observations on male British doctors. BMJ 2004; 328: 1529-33.

15.- PICHÓN RIVIERE A, BARDACH A, CAPORALE J, ALCARAZ A, AUGUSTOVSKI F, CACCAVO F, et al. Carga de Enfermedad atribuible al Tabaquismo en Chile. Documento Técnico IECS N ${ }^{\circ}$ 8. Instituto de Efectividad Clínica y Sanitaria, Buenos Aires, Argentina. Abril de 2014

16.- RIESCO MIRANDA JA. La SEPAR: determinación y compromiso de una sociedad científica ante la epidemia del tabaquismo. Arch Bronconeumol 2016; 52: 409-10.

17.- FIORE MC, GOPLERUD E, SCHROEDER SA. The Joint Commission's new tobacco-cessation measureswill hospitals do the right thing? N Engl J Med. 2012; 366: 1172-4. 